\title{
On Molecular Gelation Mechanism of L-Cysteine Based Hydrogel
}

\author{
Pavel Komarov ${ }^{1,2, *}$, Maxim Ovchinnikov ${ }^{3}$, Svetlana Khizhnyak ${ }^{2}$, Vladimir Alekseev $^{2}$, Ivan Mikhailov ${ }^{2}$, \\ Pavel Pakhomov ${ }^{2}$ \\ ${ }^{1}$ Nesmeyanov Institute of Organoelement Compounds, Russian Academy of Sciences, Vavilova st. 28, Moscow, 119991 Russia \\ ${ }^{2}$ Tver State University, Sadovyi per. 35, Tver, 170002 Russia \\ ${ }^{3}$ Tver State Medical Academy, Sovetskaya st. 4, Tver, 170642 Russia \\ *Corresponding Author: pv_komarov@mail.ru
}

Copyright (C) 2013 Horizon Research Publishing All rights reserved.

\begin{abstract}
A method for preparing a supramolecular hydrogel composed of a mixture of L-cysteine, and silver nitrate is reported. The main features of the hydrogel are gelation at low content of the dispersed phase $(\sim 0.01 \%)$ induced by addition of various salts (sulfates, sulfites, chlorides, bromides, and others) and thixotropic behavior. On the basis of experimental data, the mechanism of gelation is suggested. It is assumed that filament-like aggregates in the system are formed from silver mercaptide zwitterions due to noncovalent bonding as a result of self-assembly processes, addition of a salt leads to physical cross-linking, and gel network formation. An atomistic computer model is developed to confirm the supposed mechanism of gelation. It is established that resulting molecular configurations are stabilized by the interactions of $-\mathrm{NH}_{3}{ }^{+}$and $-\mathrm{C}(\mathrm{O}) \mathrm{O}^{-}$groups of silver mercaptide zwitterions comprising neighboring clusters. These assumptions are taken as a basis of a mesoscopic model to study the processes of nucleation and growth of filament-like aggregates on a large spatial scale.
\end{abstract}

Keywords Physical Gel, Cysteine-silver Solution, Self-assembly, Gelation, Computer Simulations

\section{Introduction}

Compounds capable of forming physical gels comprise clays, polymers, proteins, and certain small organic molecules (with molecular weight below 2000) called low-molecular-weight gelators [1], which include some substances producing hydrogels (HGs) [2,3]. The latter are of particular interest because they are widely used for the manufacture of personal care products, foodstuffs, and in recent time, have found various biomedical applications [4].

Numerous results on the phase behavior, morphology, and rheological properties of HGs are obtained [2-4]. Theoretical studies of HGs put forward two interrelated questions: (i) how and under what conditions do three-dimensional gel networks arise? and (ii) what is the mechanism of this process? The first question which has been well reported in publications [5-11] is associated with the study of critical phenomena and phase transitions that reflect the macroscopic properties of colloidal systems. The second question (the most complex and poorly studied) is related to the features of the molecular structure of HGs. This issue attracts additional interest because HGs are relatively simple and convenient systems to investigate the processes of selfassembly, and so understanding of them will open a way to the target-directed design of novel materials $[2,12]$.

In the past decade, many works concerning phenomenological models of the self-assembly of small molecules that form aggregates of extended shape such as rods, tapes, helices, tubes, and filaments were published $[1-3,12]$. When these aggregates become rather long and entangled, they transform into three-dimensional networks of different topologies, that bind solvent molecules, and depending on reagent concentration, temperature, nature of the solvent, presence of specific additives can form gels or emulsions [1-3]. Although the structural features of aggregates comprising the spatial network of gel are not studied at the molecular level, the following factors are considered to be responsible for network appearance: (i) the presence of chiral centers in molecules, (ii) van der Waals interaction, (iii) the presence of oppositely charged functional groups, (iv) hydrogen bonding ability, (v) $\pi-\pi$ stacking interaction between benzene rings, (vi) formation of polymeric metal complexes due to the donor-acceptor interactions $[1,2,13,14]$. In other words, the presence of several force centers in molecules allowing them to form coordination bonds of head-to-tail type is the main driving force for the growth of supramolecular filament-like and network aggregates in solutions of low-molecular-weight compounds.

Aggregates forming a spatial network of physical gel show an analogy with polymers; therefore, a term supramolecular polymer was introduced into scientific usage [15]. In contrast to macromolecules resulting from covalent 
bonds between monomer units, structural units in supramolecular polymers are linked by weak noncovalent intermolecular interactions of the aforementioned types.

Hydrogel based on L-cysteine and $\mathrm{AgNO}_{3}$ solutions (CSS) that we obtained recently may be also considered as a supramolecular polymer [16]. Cysteine-silver solution can form a gel at a low concentration of the initial components $(\sim 0.01 \%)$. This gel is a physical gel because it shows distinct thixotropic behavior. It should be noted that silver is a good antiseptic, while cysteine plays a key role in metabolism and provides a protective function of the binding of toxic heavy metals in a living organism. Thus, low-concentrated cysteine-silver nitrate aqueous solutions and $\mathrm{HG}$ can serve as a matrix for designing novel bioactive substances, different pharmaceuticals, and personal-care products. Moreover, CSS is a convenient model system to study selfassembly and gelation processes.

Although the complexation of L-cysteine with metal ions, including silver ions, is well studied [17], L-cysteine as gelator was previously unknown. From a classification viewpoint, L-cysteine based $\mathrm{HGs}$ are referred to as supramolecular metal-complex gels [18-20]. In this paper, the results of our studies on the supramolecular organization of CSS obtained by experimental and computer modeling techniques are presented. The experimental data (see Part 2 and 3 of this paper) allowed us to formulate the phenomenological model of gelation (Part 4); on its basis, we have built a large-scale computer model to study the gelation mechanism. Each of these tasks was sequentially solved based on two interrelated models: atomistic (Part 5) and mesoscopic (Part 6) that were developed using molecular dynamics (MD) and Monte Carlo (MC) methods, respectively.

\section{Method for Preparing Hydrogel Based on Aqueous Solutions of Cysteine and Silver Nitrate}

All chemicals for the preparation of the CSS and hydrogels were obtained from "Across" (99.8\%) and used without further purification. Solutions of the L-cysteine, $\mathrm{AgNO}_{3}$, and a salt $\left(\mathrm{Na}_{2} \mathrm{SO}_{4}\right)$ inducing the gelation were prepared with bidistilled water. It should be noted that there are a variety of salts containing single $\left(\mathrm{Cl}^{-}, \mathrm{Br}^{-}, \mathrm{HSO}_{4}^{-}\right.$, $\left.\mathrm{SCN}^{-}, \mathrm{JO}_{3}{ }^{-}, \mathrm{JO}_{4}{ }^{-}\right)$and double charged anions $\left(\mathrm{SO}_{4}{ }^{2-}, \mathrm{SO}_{3}{ }^{2-}\right.$, $\mathrm{S}_{2} \mathrm{O}_{8}{ }^{2-}, \mathrm{WO}_{4}{ }^{2-}, \mathrm{MoO}_{4}{ }^{2-}$ ) which can induce the gelation [21].

Fig. 1 demonstrates a scheme of the preparation of L-cysteine based HG [16]. Mixing of the initial components with concentrations of 3.08 and $3.85 \mathrm{mM}$, respectively, leads to the formation of a turbid sample at $\mathrm{pH} \sim 4.9$ (Fig. $1 \mathrm{~A})$, which after aging in thermostat at temperatures of 10 to $60^{\circ} \mathrm{C}$ for 0.3 - 48 hours becomes transparent and slightly yellow at $\mathrm{pH}=3.1$ (Fig. 1B). Gelation in the system starts, if a salt with the concentration one order of magnitude lower than the total content of L-cysteine and $\mathrm{AgNO}_{3}$ is added to CSS. For example, the concentration of sodium sulfate in the sample shown in Fig. 1 is $0.2 \mathrm{mM}$. The hydrogel obtained is stable in an inverted test tube (Fig. 1C).

FTIR spectroscopic study of the samples was performed on "Equinox 55" spectrometer ("Bruker"). The CSS and hydrogel samples for the measurement were frozen-dried and pressed with $\mathrm{KBr}$ into a pellet. The UV-vis spectra of the CSS was recorded on a "Specord M-40" spectrophotometer ("Carl Zeiss") in a quartz cuvette of 1 mm thickness.

Rheological studies of the hydrogels were carried out on a rotational viscometer "Carri-Med" between two plates at a fixed oscillation regime $(1 \mathrm{~Hz})$. To avoid evaporation of the solvent during the measurement, the sample was covered by a thin layer of mineral oil. Dynamic light scattering (DLS) measurements were carried out on an instrument with Al-Sp 81 goniometer, digital photon correlator ALV-5000, He-Ne laser $(632.8 \mathrm{~nm})$, power $36 \mathrm{~mW}$, and a scattering angle of $90^{\circ}$. Before the measurements, the samples were centrifuged for $20 \mathrm{~min}$ at $10,000 \mathrm{rpm}$. The size distribution of aggregates over the diffusion coefficient was determined as described in the works [22,23]. TEM images of the samples were obtained on LEO 912 AB OMEGA microscope ("Carl Zeiss") in the Shared Facilities Center of the Lomonosov Moscow State University. A sample was cast on a standard copper grid with a polymeric support film made from Formvar (polyvinylformal) about $100 \mathrm{~nm}$ thick.
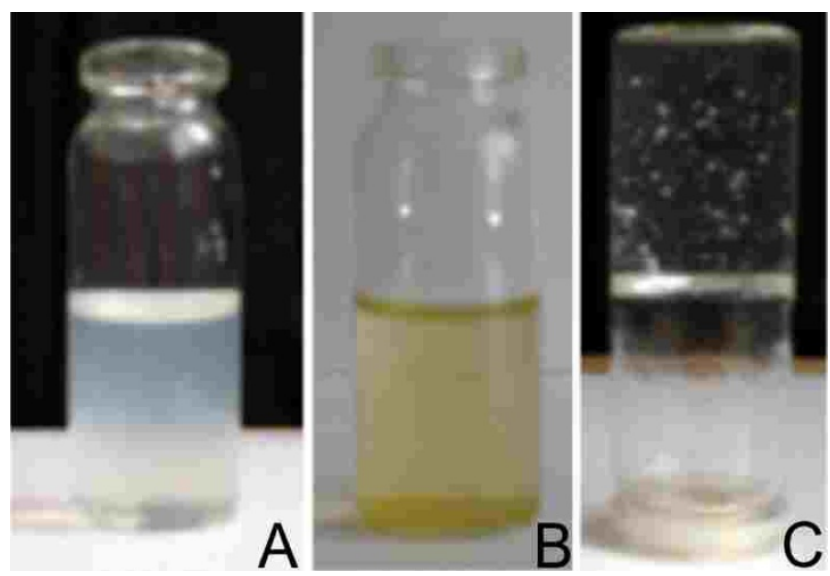

Figure 1. The scheme of the formation of supramolecular hydrogel based on L-cysteine and silver nitrate: (A) a mixture of the initial components immediately after mixing ( $\mathrm{pH} \sim 4.9)$, (B) CSS after aging for one hour at 50 ${ }^{\circ} \mathrm{C}(\mathrm{pH}=3.1),(\mathrm{C})$ a gel formed after addition of sodium sulfate.

Concentrations of L-cysteine, silver nitrate, and sodium sulfate are 3.08, 3.85 and $0.2 \mathrm{mM}$, respectively.

\section{Results of Experimental Study}

It is established that mixing of L-cysteine and $\mathrm{AgNO}_{3}$ solutions of definite concentrations and molar ratio leads to the formation of silver mercaptide (SM) [24], which is confirmed by FTIR spectroscopy. Therefore, an absorption band at $2544 \mathrm{~cm}^{-1}$ in IR spectrum of L-cysteine 
corresponding to the stretching vibrations of the thiol group (-SH) is not observed in the spectra of the CSS and the hydrogel (Fig. 2A). Moreover, it is clear that $-\mathrm{C}(\mathrm{O}) \mathrm{OH}$ groups in the studied samples are deprotonated, since the carbonyl group absorption (1710-1790 $\mathrm{cm}^{-1}$ ) is absent in the spectra (Fig. 2B) [25]. Hydrogen bonding produces significant band broadening and lowering of the mean absorption frequencies, which is more pronounced in IR spectra of the CSS and the gel. Sulfate ions in the hydrogel spectrum is observed in the range of $1130-1080 \mathrm{~cm}^{-1}$, that can be an additional confirmation of non-colavent cross-linking appearing after addition of the salt. According to the UV-vis spectroscopic data, the aging process is accompanied by the appearance and growth of the absorption bands at 310 and $390 \mathrm{~nm}$ (Fig. 3). It is assumed that the two absorption bands associated with the charge transfer between donor sulfur atoms and acceptor silver atoms of different SM particles correspond to the formation of supramolecular oligomers based on SM molecules of the following types: $\cdot \mathrm{Ag}-\mathrm{SR} \cdot \mathrm{Ag}-\mathrm{SR} \cdot \mathrm{Ag}-\mathrm{SR} \cdot$ and $\cdot \mathrm{Ag}-\mathrm{SR} \cdot \mathrm{Ag}+\cdot \mathrm{SR}-\mathrm{Ag} \cdot$, where $\mathrm{R}=\mathrm{CH}_{2} \mathrm{CH}\left(\mathrm{NH}_{2}\right) \mathrm{COOH}$. In fact, SM molecules in aqueous solution are in the zwitterionic form, which is a dipole with oppositely charged ends. Due to the electrostatic interaction of these dipoles, supramolecular oligomeric chains are formed in the CSS and, moreover, they are associated via the excess silver ions. It is found out that dilution of the CSS leads to decreasing of the absorption of the bands (310 and $390 \mathrm{~nm}$ ), that is an evidence of dissociation of the linked oligomeric chains. The presence of these bands could not be explained by plasmon resonance at silver nanoparticles, which is observed at wavelength of $400 \mathrm{~nm}$ or above depending on their shape and size [26,27].
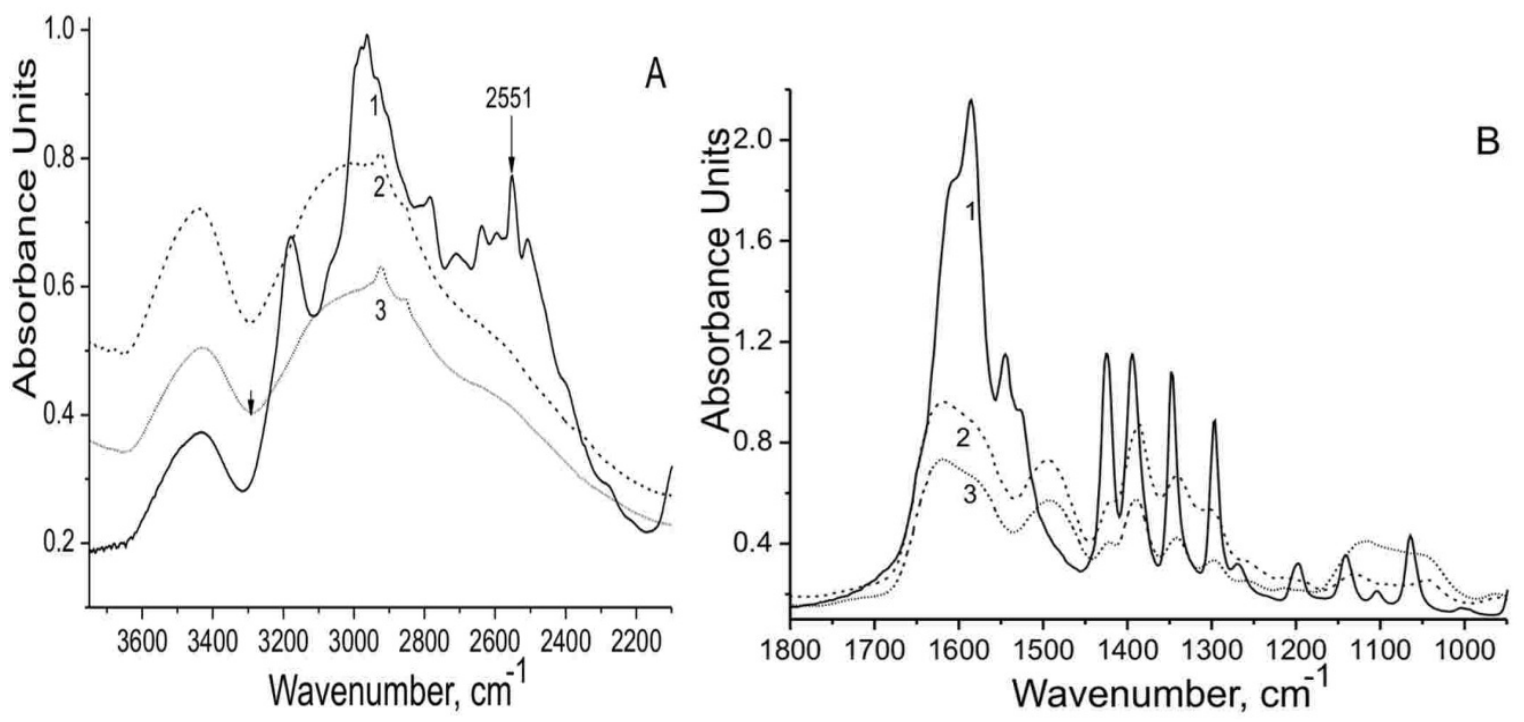

Figure 2. FTIR spectra of L-cysteine powder (1), frozen-dried CSS (2) and gel (3) in different ranges: $3750-2110 \mathrm{~cm}^{1}(\mathrm{~A}), 1800-900 \mathrm{~cm}^{-1}(\mathrm{~B})$.

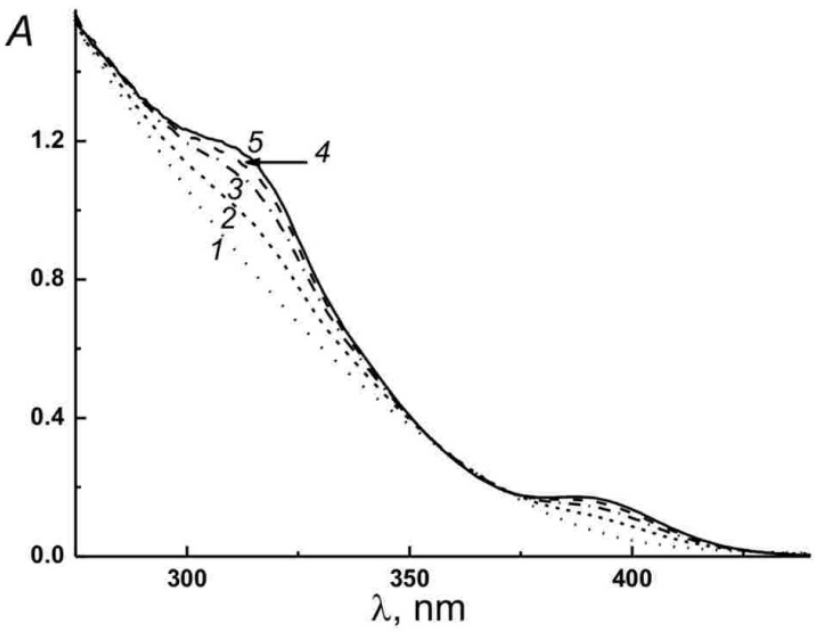

Figure 3. UV-vis absorption spectra of the CSS during the aging stage: (1) 0 , (2) 30 , (3) 60 , (4) 90 , and (5) $120 \mathrm{~min}$ after mixing of the components. $T=$ $298.15 \mathrm{~K}\left(C_{\mathrm{L}-\mathrm{Cys}}=3.0 ; C_{\mathrm{AgNO} 3}=3.75 \mathrm{mM}\right)$.

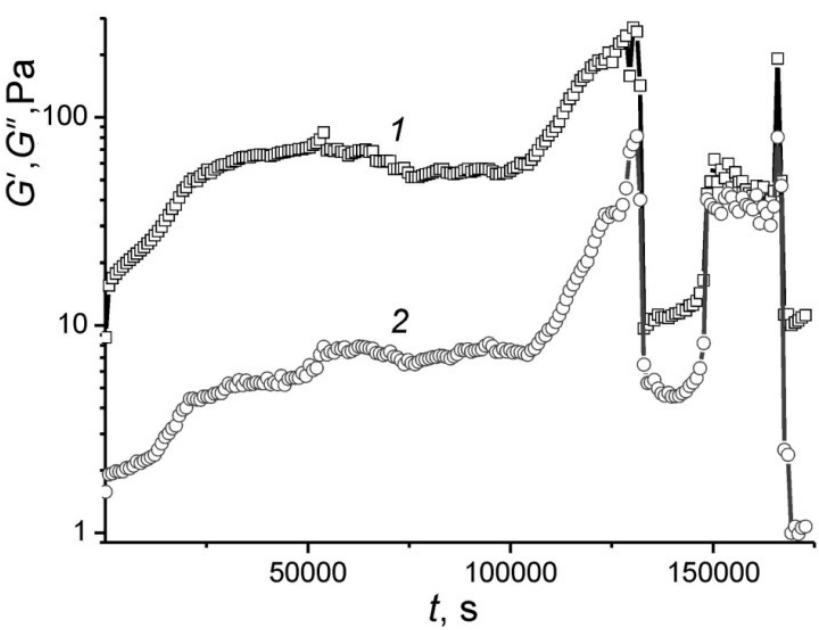

Figure 4. Time dependence of (1) storage $G$ ' and (2) loss $G$ '" modulus of $\mathrm{L}$-cysteine based gel. Gel formation induced by $\mathrm{Na}_{2} \mathrm{SO}_{4} . C_{\mathrm{L}-\mathrm{Cys}}=3.0, C_{\mathrm{AgNO}}$ $=3.75, C_{\mathrm{Na} 2 \mathrm{SO} 4}=0.4 \mathrm{mM}, T=298.15 \mathrm{~K}$, oscillation frequency $-1 \mathrm{~Hz}$, strain $-2 \%$. 
The rheological studies of prepared hydrogels showed that they have true thixotropic properties. A vigorous shaking of a test tube with a gel sample transforms it to a fluid state, whereas the gel is formed again, if the tube is left to rest. Fig. 4 characterizes the rheological behavior of the hydrogel indicating that the addition of the salt in the CSS causes in time very slow growth of the storage $\left(G^{\prime}\right)$ and loss $\left(G^{\prime \prime}\right)$ modulus values. Then, a sharp increase and decrease of the modulus is observed, when a certain critical value of $t$ is reached. The further keeping of the hydrogel in a weakly oscillating mode resulted in a repeated drastic growth and abrupt drop of the modulus. Such a rheological behavior is typical for thixotropic systems [30]. One can suppose that the addition of a salt to the CSS gives rise to a spatial network formation (the solution becomes a gel). However, this network is weak because of non-covalent character of cross-linking, and therefore, it readily breaks down under the action of shear stress in a rheometer.

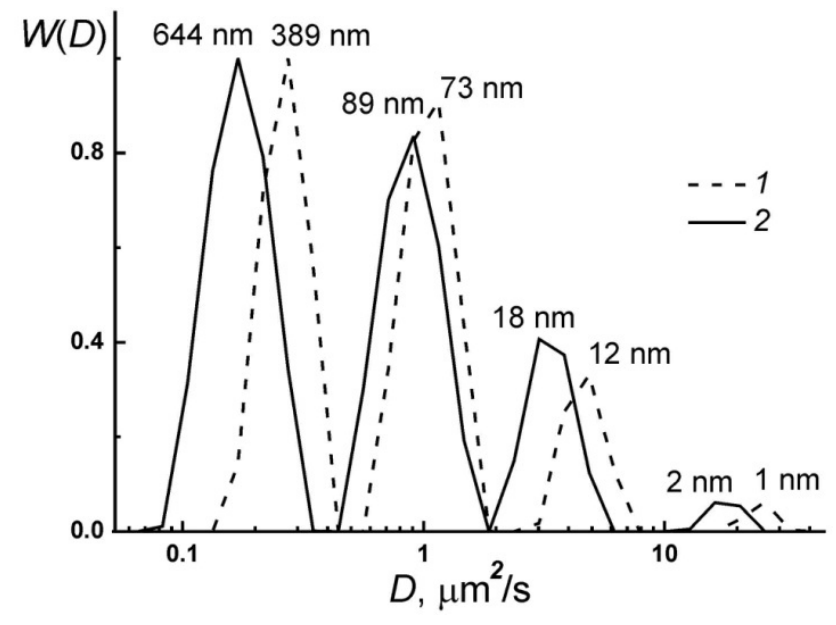

Figure 5. Diffusion coefficient distribution for CSS at different time of storage: $(1)-30,(2)-122 \min \left(C_{\mathrm{L}-\mathrm{cys}}=2.7 \mathrm{mM}, C_{\mathrm{AgNO} 3}=3.3 \mathrm{mM}\right)$.

The study of CSS by DLS showed the presence of a set of clusters of different sizes (Fig. 5), which are increased with time. It was established that the clusters in CSS have a positive charge that agrees well with the data of potentiometric measurements. Fig. 6 shows the TEM images of the solutions at different concentrations (Fig. 6A and Fig. 6B) and the hydrogel at different magnifications (Fig. 6C and Fig. 6D). It is seen from the figure that the CSS is composed of separate clusters, but as it can be seen from electronogram doesn't contain nanoparticles (see inset, Fig. 6A). Filament-like intersecting structures composed of the same clusters are observed in the gel (Fig. 6C). The structure and density of the three-dimensional network depends on the type of salt and concentration of the initial components [21]. Fig. 6D illustrates a gel network with a fiber-like structure resulting from the addition of $\mathrm{Na}_{2} \mathrm{SO}_{4}$ into CSS. We established that the network structure also appears in highly diluted CSS without addition of a salt (Fig. 6B).

Thus, as a result of the performed experimental study, we established that the system under consideration tends to self-assembly, and forms filament-like structures and a strongly branched gel network. It is important to note that such a structure of gel network provides a good explanation for the fact that such a small quantity of dissolved substance $(\leq 0.01 \%)$ can form the gel at a relatively large amount of water.

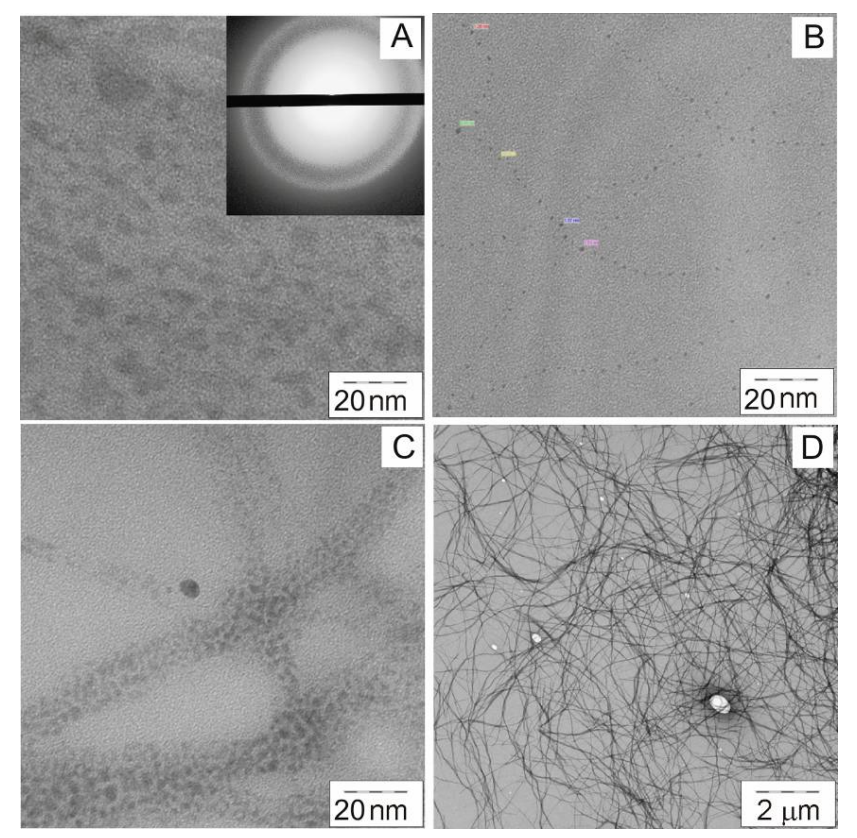

Figure 6. TEM images of: (A) - CSS $(0.07 \%)$ and the corresponding electronogram (inset), (B) - highly diluted CSS (0,005\%); (C) and (D) $\mathrm{L}$-cysteine based hydrogel at various magnifications $\left(\mathrm{CNa}_{2} \mathrm{SO}_{4}=0.8 \mathrm{mM}\right)$.

\section{Phenomenological Model of Gelation in CSS}

Let us consider the main reasons that can cause gelation in CSS. Cysteine carries three functional groups namely $-\mathrm{NH}_{2},-\mathrm{C}(\mathrm{O}) \mathrm{OH}$, and $-\mathrm{SH}$ which are capable of coordinating with metal ions and producing metal complexes. This property of cysteine is used for the preparation of $\mathrm{Ag}$ and metal sulfides nanoparticles [31-37]. Cysteine molecules are considered as a sulfur source for the sulfide formation and as a stabilizer of resulting nanoparticles. Since the concentrations of reagents used in CSS is within the range where $\mathrm{Ag}$ thiolate complexes prevail (on account of the high affinity of thiols to silver, for cysteine $\log K f=11.9$ ) [38], all silver ions are coordinated to the thiol groups to form silver mercaptide (SM) (Fig. 7A) followed by the formation of more complex aggregates, silver mercaptide clusters (Fig. 7B).

Due to the presence of oppositely charged functional groups, SM clusters in the closely arranged aggregates are able to interact, forming hydrogen bonds between $-\mathrm{C}(\mathrm{O}) \mathrm{O}^{-}$ and $-\mathrm{NH}^{+}$groups, which can induce the self-assembly of SM aggregates [33,35,36]. The authors of the work [33] also reported evidence for the possibility of aggregation of 
colloidal particles due to hydrogen bonding between the functional groups of cysteine molecules attached to the different particles of colloidal silver. Indirect evidence for the self-assembly of resulting clusters via $-\mathrm{C}(\mathrm{O}) \mathrm{O}^{-}$and $-\mathrm{NH}_{3}{ }^{+}$groups is provided by the fact that hydrogels could not be obtained from cysteamine (which has no carboxyl groups in contrast to cysteine) or mercaptoethanol (which has no carboxyl and amino groups), whereas thiol (-SH) groups are present in these compounds.

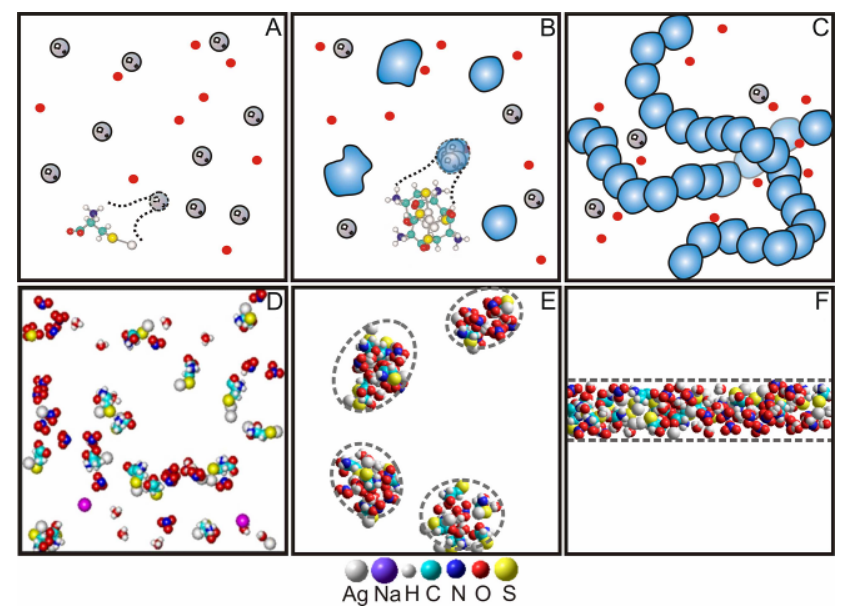

Figure 7. Main stages of the gelation: (A) the formation of the SM from L-cysteine and silver nitrate molecules; (B) ageing of CSS; (C) the gel network formation. Small and middle circles indicate the low- molecularweight ions and silver mercaptide molecules, respectively, and large irregular shapes correspond to the clusters of SM particles. The initial states of the atomistic model which correspond to the main stages of the CSS gelation: (D) a disordered; (E) a cluster; (F) a filament-like. The dashed lines indicate the prescribed templates for SM particles.

As stated above, the data of the potentiometric analysis indicate that SM in CSS exists in the form of zwitterions. The positive charge in the clusters (which impart stability to the system) arises due to coordination of excess silver ions to thiol groups. It is obvious that the subsequent addition of sodium sulfate disturbs the stability of CSS, which may result in the gelation in the solution on account of the formation of hydrogen bonds between the $-\mathrm{C}(\mathrm{O}) \mathrm{O}^{-}$and $-\mathrm{NH}^{+}$groups of neighboring SM clusters (Fig. 7C). Such processes of aggregative instability are well-known in colloidal chemistry [39]. In terms of the effect of electrolyte additives on the coagulation in colloidal dispersions, CSS may be ascribed to the lyophobic colloids.

Thus, we can conclude that self-assembly of CSS can be divided into three main stages schematically represented in Fig. 7, namely, (i) the formation of silver mercaptide (Fig. 7A), (ii) the aggregation of SM into small clusters (Fig. 7B), and (iii) the subsequent self-assembly of the clusters to form a gel network (Fig. 7C).

\section{Atomistic Simulation of CSS}

\subsection{Main Parameters of the Model}

To confirm the proposed mechanism of gelation in CSS, we have developed an atomistic computer model. We suppose that the process of SM formation is completed, and $\mathrm{Na}_{2} \mathrm{SO}_{4}$ which induces gelation is already added in the aged solution. Taking into account the data of the quantitative analysis of CSS (described above), the following components were introduced into the model: silver mercaptide in the form of zwitterions, $\mathrm{NO}_{3}^{-}, \mathrm{SO}_{4}{ }^{2-}, \mathrm{Ag}^{+}$, $\mathrm{Na}^{+}$, and $\mathrm{H}_{3} \mathrm{O}^{+}$ions, and water molecules. These ions result from the formation of SM zwitterions, and dissociation of $\mathrm{AgNO}_{3}$ and $\mathrm{Na}_{2} \mathrm{SO}_{4}$ salts. Thus, the total number of subsystems in the model is 7 .

The number of components in each subsystem is determined by the following parameters: $N$ is the number of zwitterions, $N_{\mathrm{NO} 3}, N_{\mathrm{Ag}}, N_{\mathrm{H} 30}, N_{\mathrm{SO} 4}$, and $N_{\mathrm{Na}}$ are the numbers of $\mathrm{NO}_{3}^{-}, \mathrm{Ag}^{+}, \mathrm{H}_{3} \mathrm{O}+, \mathrm{SO}_{4}^{2-}$, and $\mathrm{Na}^{+}$ions, respectively. There is a simple relationship between these parameters. It is supposed that one SM particle is formed from one cysteine molecule and one silver nitrate molecule in the presence of an excess of silver nitrate. The excess of $\mathrm{AgNO}_{3}$ may be specified on the basis of the concentrations of 3.08, 3.85, and $0.2 \mathrm{mM}$ for L-cysteine, silver nitrate, and sodium sulfate, respectively, which we use to obtain the gel sample shown in Fig. 1. Taking into account these values, we suppose that $N_{\mathrm{NO} 3}=4 N / 3$ and $N_{\mathrm{Ag}}=N / 3$. Since $\mathrm{H}_{3} \mathrm{O}^{+}$ ions result from the dissociation of $-\mathrm{SH}$ and $-\mathrm{C}(\mathrm{O}) \mathrm{OH}$ groups followed by the formation of SM zwitterions, their number $N_{\mathrm{H} 3 \mathrm{O}}=N$. The number of $\mathrm{SO}_{4}{ }^{2-}$ and $\mathrm{Na}^{+}$ions was assigned as $N_{\mathrm{SO} 4}=N_{\mathrm{NO} 3} / 20$ and $N_{\mathrm{Na}}=N_{\mathrm{NO} 3} / 10$. Thus, to determine the quantitative composition of all subsystems, it is sufficient to assign the total number of SM zwitterions to be $N$. Let us note that the total charge in the model is zero.

\subsection{Parameterization of Valence Force Field}

To calculate intra- and intermolecular forces, we used an expression for the total potential energy in a functional representation used in the valence force field (VFF) AMBER [40,41]:

$$
\begin{aligned}
& V\left(\mathbf{r}_{1} \mathbf{r}_{2} \ldots \mathbf{r}_{N}\right)=\sum_{\text {bonds }} \frac{1}{2} k_{i j}\left(l_{i j}-l_{i j, 0}\right)^{2}+ \\
& +\sum_{\text {angles }} \frac{1}{2} k_{i j k}\left(\theta_{i j k}-\theta_{i j k, 0}\right)^{2}+\sum_{i m p r o p e r} \frac{1}{2} k_{i j k l}^{i m p}\left(\omega_{i j k l}-\omega_{i j k l, 0}\right)^{2}+ \\
& +\sum_{\text {torsions }} \frac{1}{2} k_{i j k l} \text { prop }\left(1+\cos \left(n_{i j k l} \omega_{i j k l}-\gamma_{i j k l}\right)\right)+ \\
& +\sum_{j=1}^{N-1} \sum_{i=j+1}^{N} \sqrt{\varepsilon_{i} \varepsilon_{j}}\left[\left(\frac{\sigma_{i}+\sigma_{j}}{2 r_{i j}}\right)^{12}-2\left(\frac{\sigma_{i}+\sigma_{j}}{2 r_{i j}}\right)^{6}\right]+ \\
& +\sum_{j=1}^{N-1} \sum_{i=j+1}^{N} \frac{q_{i} q_{j}}{4 \pi \varepsilon_{0} r_{i j}}
\end{aligned}
$$

where $l_{\mathrm{ij}}=\left|\mathbf{r}_{\mathrm{ij}}\right|$ is the distance between the selected pair of atoms, the summands describe the energy contribution of 
valence bond deformation, valence angles $\theta_{\mathrm{ijk}}$, dihedral and improper dihedral angles $\omega_{\mathrm{ijk} k}$, van der Waals and electrostatic interaction, respectively.

The values of constants $k_{\mathrm{ij}}, l_{\mathrm{ij}, 0}, k_{\mathrm{ijk}}, \theta_{\mathrm{ijk}, 0}, k_{\mathrm{ijkl}}$ imp,$\omega_{\mathrm{ijkl}, 0}$, $k_{\mathrm{ijkl}}^{\text {prop }}, n_{\mathrm{ijk}}, \gamma_{\mathrm{ijkl}}, \varepsilon_{\mathrm{i}}$, and $\sigma_{\mathrm{i}}$, except for cases when silver is one of the interacting atoms, and for $\mathrm{NO}_{3}^{-}, \mathrm{SO}_{4}{ }^{2-}$ ions, were taken from VFF AMBER 10 [41]. For water molecules, we used the TIP3P model representation [41,42]. The parameterization of $\mathrm{Ag}^{+}, \mathrm{NO}_{3}{ }^{-}, \mathrm{SO}_{4}{ }^{2-}$ ions was accomplished using VFF PCFF [43]. Force constants for potentials viz. the stretching of Ag-S valence bond is $k_{\mathrm{AgS}}=1520 \mathrm{kcal} /\left(\mathrm{mol} \cdot \AA^{2}\right)$ and $l_{\mathrm{AgS}, 0}=2.4 \AA$; the deformation of Ag-S-C valence angles $k_{\mathrm{AgSC}}=540 \mathrm{kcal} /\left(\mathrm{mol} \cdot \mathrm{rad}^{2}\right)$ and $l_{\mathrm{AgSC}, 0}=70 \mathrm{deg}$; and the deformation of Ag-S-C-C and Ag-S-C-H torsion angle $k_{\mathrm{AgSCC}}{ }^{\mathrm{prop}}=0.5 \mathrm{kcal} / \mathrm{mol}, n_{\mathrm{AgSCC}}=2, \gamma_{\mathrm{AgSCC}}=150 \mathrm{deg}$, and $k_{\mathrm{AgSCH}}{ }^{\text {prop }}=2.0 \mathrm{kcal} / \mathrm{mol}, n_{\mathrm{AgSCH}}=3, \gamma_{\mathrm{AgSCH}}=130 \mathrm{deg}$. These parameters and partial charges on atoms were calculated within the semiempirical method of molecular orbitals ZINDO/1 [44] with the use of the Hyperchem ${ }^{\mathrm{TM}} 8.0 .8$ software package [45], allowing the modeling of compounds containing transition metals.

\subsection{Noncovalent Interaction of Sulfur \& Silver Atoms}

To take into account the possibility of the formation of intermolecular bonds between SM particles due to the sulfur-argentum noncovalent interactions, we additionally introduced in the model for SM two unshared electron pairs (EP) at sulfur atoms. The energetic parameters for the deformation of covalent bonds, bond angles, and torsion angles for EP are involved in VFF AMBER 10. The absent parameters for constants $\varepsilon_{\mathrm{EPAg}}=\left(\varepsilon_{\mathrm{EP}} \varepsilon_{\mathrm{Ag}}\right)^{1 / 2}$ and $\sigma_{\mathrm{EPAg}}=\left(\sigma_{\mathrm{EP}}\right.$ $\left.+\sigma_{\mathrm{Ag}}\right) / 2$ in (1) were calculated by the ZINDO/1 method and in the Hartree-Fock approximation with the $3-21 \mathrm{G}$ basis set (using the Hyperchem ${ }^{\mathrm{TM}}$ 8.0.8 software package). The following assessments of the constants were obtained: 23.01 $\mathrm{kcal} / \mathrm{mol}(\mathrm{ZINDO} / 1)$ and $104.99 \mathrm{kcal} / \mathrm{mol}(3-21 \mathrm{G})$ for $\varepsilon_{\mathrm{EPAg}}$, $2.68 \AA(Z I N D O / 1)$ and $2.53 \AA(3-21 G)$ for $\sigma_{\text {EPAg }}$. Since the $\sigma_{\text {EPAg }}$ parameter calculated by two methods has close values, we used the average value of $2.61 \AA$ for CSS modeling. The magnitude of energetic parameter, $\varepsilon_{\mathrm{EPAg}}$, was refined by the modeling of dimers of SM particles surrounded by 20 water molecules under conditions of NVT ensemble by the molecular dynamics method. The methodology of molecular dynamics calculations will be described below. Silver mercaptide particles formed the dimer via -S-EP $\cdots$ Ag-S- interactions. The value of $\varepsilon_{\mathrm{EPAg}}=44.72$ $\mathrm{kcal} / \mathrm{mol}$ was selected in such a manner that the dimer is decomposed at a temperature above $340 \mathrm{~K}$ [46].

\subsection{The Preparation of Initial States of CSS}

To study gelation in CSS we consider three different types of initial states (IS) of the computer model: (i) a disordered state, when all the molecules are randomly distributed; (ii) a cluster state, when the simulation cell contains 3-4 clusters of SM; and (iii) a filament-like state, when the simulation cell contains one filament-like aggregate (see Fig. 7D, Fig. 7E and Fig. 7F). They are supposed to correspond to different experimentally observed stages of the self-assembly of CSS schematically represented in Fig. 7A, Fig. 7B, and Fig. 7C, namely, the completion of formation of the SM molecules, the aging of the solution, and the gel-state.

The first type of initial state corresponds to the direct problem, when the system evolution begins with the randomly generated configuration of molecules and caused only by the features of intermolecular interaction. The second and the third types of IS may be coupled with the so-called inverse problem when the evolution of preliminary prepared several clusters or one filament-like aggregates is studied. The clusters aggregates are considered to be an intermediate stage of system evolution during the formation of the spatial network of gel from the disordered state. Since the clusters and filament-like aggregates are observed in the real CSS, the inverse problem allows one to overcome in part difficulties that arise in the study of self-assembly processes over fixed time intervals using atomistic models.

The modeling of the CSS evolution was carried out in a cubic cell with the edge length of $53 \AA$ where one filament-like aggregate can be formed on the base of $N=15$ SM zwitterions. To generate CSS samples with different initial ordering, a simulation cell was sequentially filled with SM zwitterions and other components with either a random-number generator or according to prescribed templates. The use of the templates supposes that some molecular subsystems are formed within domains of prescribed geometry, spherical or cylindrical, to construct several clusters or one filament-like aggregate of SM zwitterions (see Fig. 7E and Fig. 7F). Water molecules were introduced last. The number of water molecules, 4662, was selected to obtain the total density about $1 \mathrm{~g} / \mathrm{cm}^{3}$. To exclude the effect of initial configuration on the final state, we generated three statistically independent systems for the disordered and the cluster type of the initial states and six systems for the filament-like IS.

\subsection{Simulation Methodology}

The prepared samples were used to study CSS evolution at constant temperature $T=300 \mathrm{~K}$ and a volume (NVT ensemble) within the method of atomistic molecular dynamics using a DL POLY software package [47]. The integration of equations of motion was accomplished by the Verlet algorithm at 1-fs step and the cut-off radius of short-range forces of $12 \AA$. Electrostatic interaction was calculated by the Ewald summation [48] with convergence parameter $\alpha=0.345 \AA^{-1}$. Nose-Hoover thermostat was used for the renormalization of atom velocities. The dielectric constant of medium was specified to be 1 because we took into account all partial charges in the explicit form.

The primary relaxation of the models was accomplished under conditions of NVE ensemble at $T=300 \mathrm{~K}$ during 100 
ps. The velocities were renormalized at each simulation step. Then, the evolution of the CSS model was studied under conditions of NVT ( $T, V=$ const) ensemble during $5 \mathrm{~ns}$ for the disordered and the cluster initial states, and during $10 \mathrm{~ns}$ for the filament-like IS. The obtained Cartesian coordinates of atoms, that form all subsystems of the model, were saved in trajectory files every $100 \mathrm{ps}$ for the subsequent analysis of the solution evolution course. All simulations were performed with the MVS-100k cluster in the Joint Supercomputer Center, Russian Academy of Sciences.

\subsection{Results}

Fig. 8 shows the typical behavior of total energy $(E)$ of CSS model during 5 ns for three different systems which have the disordered, cluster, and filament-like initial states. The profile of $E(t)$ shows the main evolution stages of the prepared variants of CSS. It is seen in the first case that energy has a stepwise profile on account of the gradual coalescence of resulting aggregates whose average size in the final state (at the moment $t_{\max }$ ) is $\sim 10 \AA$ (Fig. 9A). For all types of initial states, the total energy at first undergoes fast change owing to the optimization of the distribution of ions and the structure of aggregates. Then, system energy is virtually constant. It is seen that the total energies of the systems with the disordered and the cluster initial states tend to converge. In the final state of these systems, cluster aggregates of elongate and branched shape are observed; see example snapshots in Fig. 9A and Fig. 9B. On average, the evolution of IS of the first type results in two aggregates, while IS of the second type produces one branched aggregate. Visual analysis shows that the mutual ordering of SM particles in the aggregates that result from the evolution of chaotic IS are similar to SM ordering resulting from the evolution of the initial cluster ordering. As a rule, two types of structures arise: clusters, and tape-like aggregates. In the first case, silver and sulfur atoms comprise compact nuclei. In the second one, neighboring zwitterions of SM are bound via interaction of their dissociated carboxyl and protonated amino groups, and sulfur-silver bonds. Thus, we can conclude that the evolution of chaotic IS at achieved modeling times leads to the formation of several clusters which can coalesce into branched structures.

The study of evolution of the filament-like IS, that supposedly have to correspond to the final state of CSS model, showed that three aggregates among six prepared systems retain the initial ordering. They are shown in Fig. 9C and Fig. 9D. One filament is transformed into an aggregate with thin contraction composed of low-molecular-weight ions (Fig. 9E), and the two remainder filaments decomposed over 1 and 1.8 ns to give extended aggregates whose structure remained virtually unchanged when modeling continued (see Fig. 9F).

The analysis of trajectory files allowed us to trace stepwise the process of evolution of filament-like aggregates in time. The decomposition of two out of six prepared filaments occurred by the same scheme. The cations and zwitterions of SM are slowly tightened to form compact groups of extended shape linked via necks composed of low-molecular-weight ions, which gradually decompose because of thermal motion. The final state shown in Fig. 9E has a characteristic narrowing composed of ions and arising in a filament-like aggregate before its breaking. Thus, one can draw a conclusion that the chains composed of one row of SM molecules are unstable as a whole, although explicit allowance made for sulfur-silver interaction stabilizes the filament-like aggregate. In other words, it is not excluded that aggregates retaining a filament-like state can degrade spontaneously over long time intervals. The supplementary justification for this fact will be discussed below.

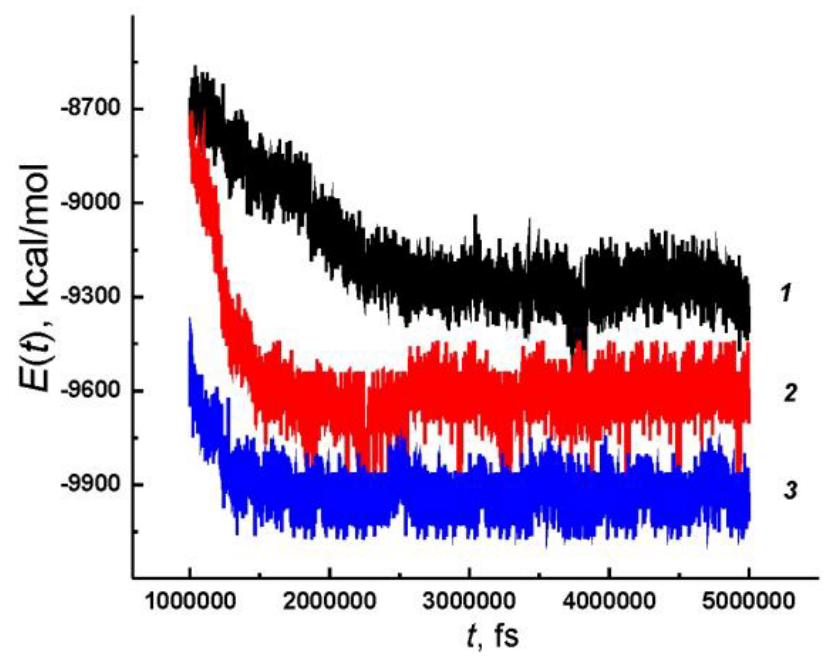

Figure 8. Time dependence for the change of the total energy $(E)$ of CSS model, $T=300 \mathrm{~K}$. (1) a system without the initial ordering, (2) a group of clusters of SM particles is present in the initial state, (3) a filament-like aggregate is present in the initial state.

The analysis of the internal structure of aggregates formed at the final state during evolution of the disordered initial state, the cluster state, and after degradation of the filament-like structures, shows that SM molecules produce cluster structures on account of numerous contiguous sulfur-silver bonds and bonds between the $-\mathrm{NH}_{3}{ }^{+}$and $-\mathrm{C}(\mathrm{O}) \mathrm{O}^{-}$groups of neighboring zwitterions of $\mathrm{SM}$. The aggregates also involve $\mathrm{SO}_{4}{ }^{2-}, \mathrm{NO}_{3}^{-}, \mathrm{H}_{3} \mathrm{O}^{+}$, and $\mathrm{Ag}^{+}$ions attracted to $-\mathrm{NH}_{3}{ }^{+}$and $-\mathrm{C}(\mathrm{O}) \mathrm{O}^{-}$groups of $\mathrm{SM}$, respectively, see Fig. 10A.

Fig. 10B shows a schematic representation of the structure of resulting chains. They constitute a chain supramolecular aggregate formed by clusters of SM particles. They are linked by contiguous bonds between neighboring clusters: (I) with participation of dissociated hydroxyl and protonated amino groups, and (II) low-molecular-weight ions coordinated by $-\mathrm{NH}_{3}{ }^{+}$, and $-\mathrm{C}(\mathrm{O}) \mathrm{O}^{-}$groups. Since $-\mathrm{NH}_{3}{ }^{+}$and $-\mathrm{C}(\mathrm{O}) \mathrm{O}^{-}$functional groups are disposed preferably on the surface of aggregates, not only chain but also branched structures can be formed in bulk solution. 


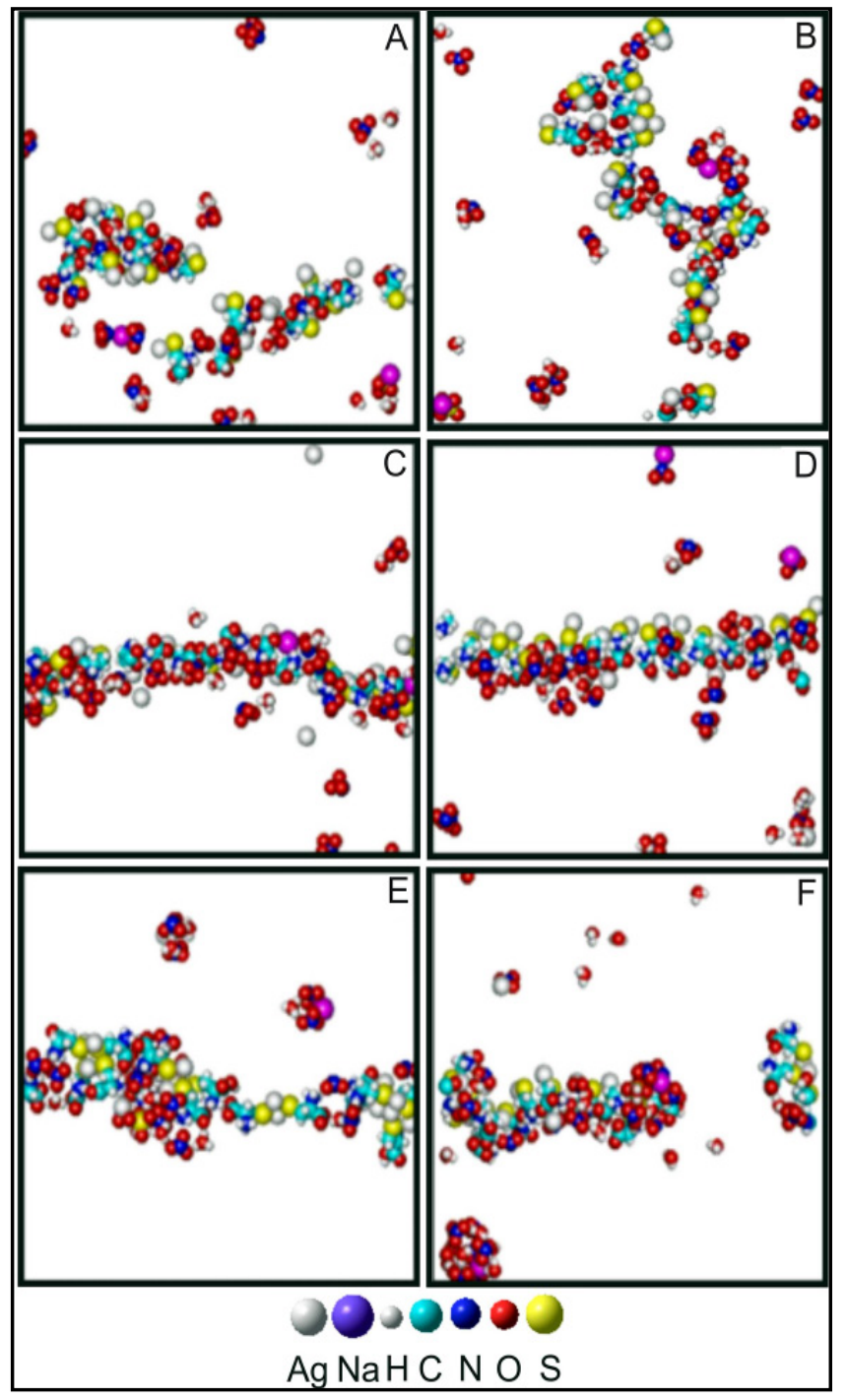

Figure 9. The final states of CSS that: (A) has no initial ordering, (B) a group of clusters is present in the initial state, $(\mathrm{C}-\mathrm{F})$ has a filament-like ordering. Water molecules are omitted for clarity, $N=15, T=300 \mathrm{~K}$.

The centers of clusters formed by SM contain sulfur and silver atoms (see Fig. 10), therefore one can suppose that the filaments of a gel network can have a similar structure. Indeed, the dots in the TEM images (Fig. 6) might be clusters and aggregates [21]. The latter is possible, if SM zwitterions in the filaments of the gel network are bundled together in such a manner that constituent sulfur and silver atoms form compact structures.

Structures similar to that shown in Fig. 9F (their average diameter is $16 \AA$ ) show a tendency to retain stability without substantial internal rearrangement for a long time. This analysis was performed based on the assessment of the average number of bonds between sulfur-silver atom pairs. The results of averaging over trajectory files show that sulfur atoms in average form $1.7 \pm 0.3$ bonds with silver atoms from neighboring molecules. In this case, the concentration of SM molecules per unit volume in such aggregates is twice as high as that in the filaments shown in Fig. 9C and Fig. 9D. This analysis allows us to assume that aggregates built from the double number of SM molecules at the constant size of the simulation cell will be stable. To verify this hypothesis, we constructed aggregates composed of two parallel chains (along the OX axis) of SM particles. The total number $N$ of SM zwitterions and cations $N$ was 30 . The size of modeling cell was $53 \AA$ as previously. As a whole, four novel variants of mutual ordering of SM particles were generated for calculations, see Fig. 11A. The morphology of the aggregates in the final state (Fig. 11B) is almost unchanged compared with the initial states, and all of them are stable at achieved modeling times. At the same time, an increase in ensemble temperature above $340 \mathrm{~K}$ leads to the fast, over 2-3 ns, gradual degradation of filaments to form several disordered clusters. The average diameter of new filaments in the resulting states is about $15.5 \AA$, i.e., it corresponds to the diameter of the aggregate shown in Fig. 9F. All filament-like aggregates, similar to Fig. 11B, comprise a chain of clusters formed by SM particles, bound to each other mainly through the interaction of $-\mathrm{NH}^{3+}$ and $-\mathrm{C}(\mathrm{O}) \mathrm{O}^{-}$groups of $\mathrm{SM}$ particles that belong to neighboring clusters (Fig. 10A, variant of cluster interaction I). In our opinion, this is the main stabilizing factor for the considered filament-like aggregates, since ion bridges (shown in Fig. 10B, interaction variant II) show a tendency to gradual degradation.

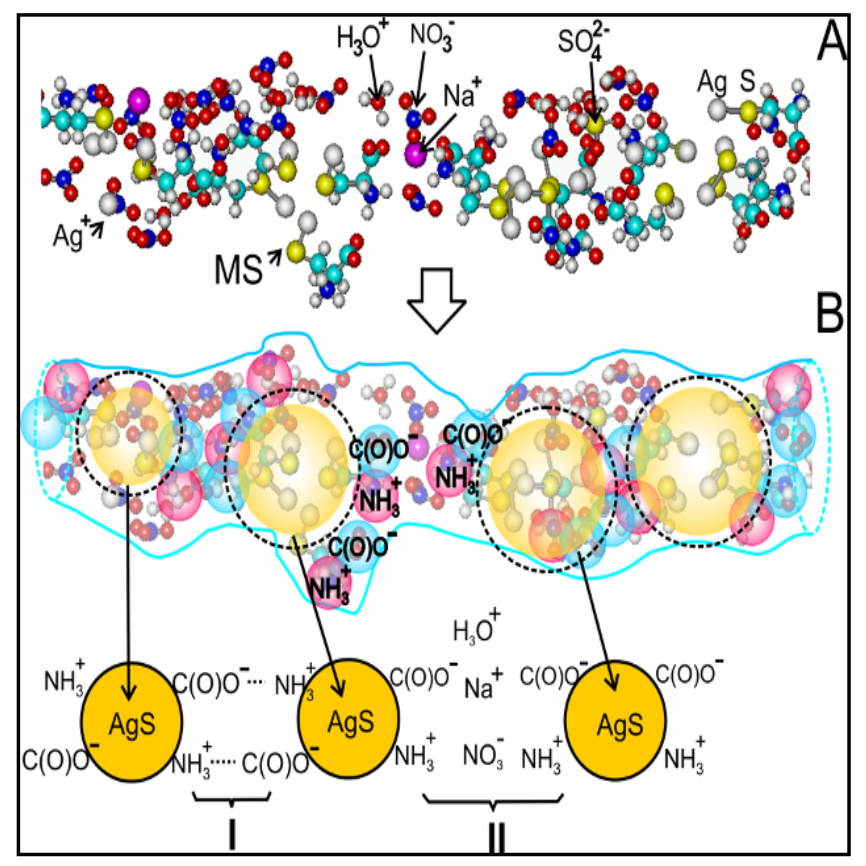

Figure 10. Typical structure of filament-like aggregates. (A) Magnified snapshot of molecular aggregate ( $\mathrm{SM}$ particles, $\mathrm{NO}_{3}{ }^{-}, \mathrm{SO}_{4}{ }^{2-}, \mathrm{H}_{3} \mathrm{O}^{+}, \mathrm{Ag}^{+}$, and $\mathrm{Na}^{+}$ions are shown). (B) Model representation of filaments comprising SM clusters bound via: (I) hydrogen bonds between $-\mathrm{NH}_{3}{ }^{+}$and $-\mathrm{C}(\mathrm{O}) \mathrm{O}^{-}$ functional groups, (II) ionic crosslinks. 


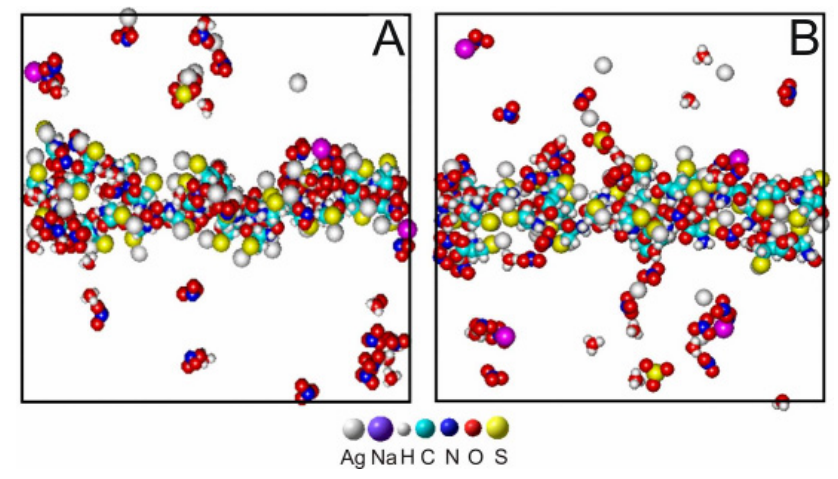

Figure 11. Snapshots of the contents of modeling cells for (A) the initial and (B) final states of filament-like aggregate with $N=30, T=300 \mathrm{~K}$, water molecules are omitted for clarity.

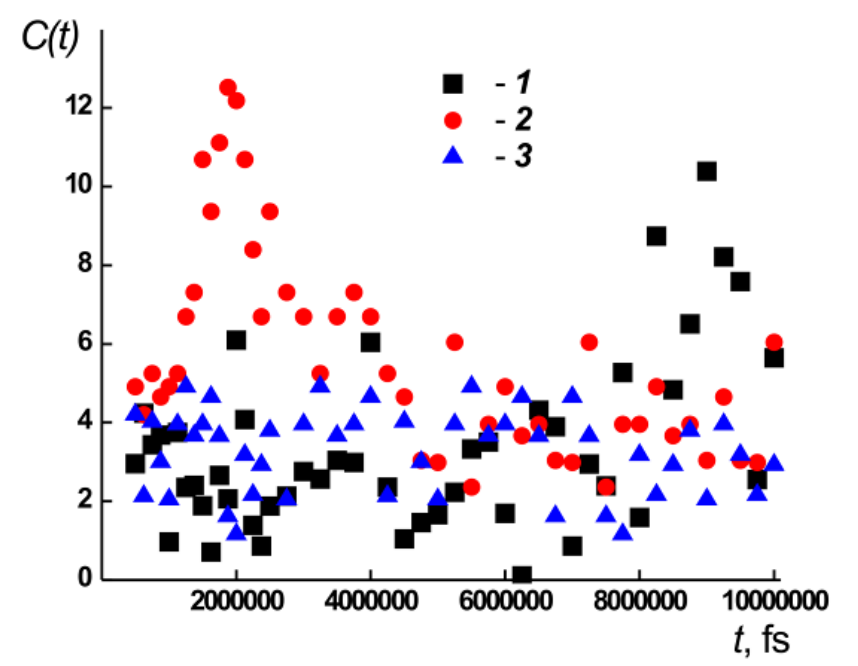

Figure 12. Time dependence for the acylindricity $(T=300 \mathrm{~K})$ of: (1) the filament-like aggregate with $N=15$ shown in Fig. 9C, (2) the filament-like aggregate with $N=15$ shown in Fig. 9F, (3) the filament-like aggregate with $N=30$ shown in Fig. 11 .

The supplementary analysis of stability of filament-like aggregates was carried out by the consideration of the behavior of their acylindricity, $c$, depending on modeling time. The value of this characteristic is determined as follows

$$
c=\left|J_{Z Z}^{2}-J_{Y Y}^{2}\right|,
$$

where $J_{\mathrm{ii}}$ are the principal moments of inertia calculated in the center-of-mass system. The inertia tensor was calculated using the atom coordinates of SM particles. The larger magnitude of acylindricity gives the higher deviation of the mutual ordering of SM particles from the state with axial symmetry. For the sake of comparison, we calculated $c(t)$ for two aggregates in the case $N=15$ and one when $N=30$. The final states of each of the selected systems are shown in Fig. 9C, Fig. 9F and Fig. 11. For the first aggregate, as it can be seen in Fig.12, the magnitude of $c(t)$ for $t<7.5 \mathrm{~ns}$ fluctuates about the average value of 2.62, then it strongly increases due to enhanced shape distortion resulting from the gradual contraction of SM into compact clusters. The similar growth of acylindricity is also observed for the second considered aggregate, and it reaches the maximum value when the filament breaks. Then $c(t)$ quickly decreases owing to the contraction of the aggregate to a cigar-shaped species shown in Fig. 9F. Its shape is virtually unchanged upon further modeling, while the magnitude of $c(t)$ fluctuates about the average value of 3.93. Thus, one can draw a conclusion that all aggregates in the case $N=15$ are unstable. For the filament-like aggregate in the case $N=30$, which retains its shape within all modeling process, the acylindricity fluctuates about the average value $\langle c\rangle=3.26$. Similar behavior for acylindricity is observed for all filament-like aggregates when $N=30$, that defines these structures as more stable as compared with aggregates in the case $N=15$.

In summary, we can conclude that the atomistic model of CSS derived from the generalization of experimental data provides an opportunity to observe a possible molecular mechanism of gel network formation. The essence of this mechanism is the following:

(i) the appearance of sulfur-silver bonds between the neighboring zwitterions of SM leads to the formation of stable clusters;

(ii) the protonated amino groups and dissociated carboxyl groups are disposed on the surface of resulting clusters;

(iii) the interaction of neighboring $\mathrm{SM}$ clusters via $-\mathrm{NH}_{3}{ }^{+}$ and $-\mathrm{C}(\mathrm{O}) \mathrm{O}^{-}$groups allows them to form filament-like aggregates.

\section{Mesoscopic Simulation of CSS}

We developed a simple coarse-grained model based on the Monte Carlo method to illustrate the formation of gel network of SM clusters on account of interaction of $-\mathrm{NH}_{3}{ }^{+}$ and $-\mathrm{C}(\mathrm{O}) \mathrm{O}^{-}$groups located on their surface. The model suggests that the process of cluster formation from SM particles is completed, while the solution of salt - initiator of the gelation has been introduced. To reduce the number of degrees of freedom, the SM clusters are considered as spherical particles of fixed diameter $\sigma_{\mathrm{MC}}$. Further, we will describe them as subsystem $A$. Uniformly distributed in solution mobile ions resulting from the dissociation of excess of $\mathrm{AgNO}_{3}$ and salt initiating gelation can be taken into account implicitly using screened Coulomb potential. The diameter of SM clusters can be calculated as $2\left(3 V_{\mathrm{MC}} N / 4 \pi\right)^{1 / 3} / 0.6$, where $V_{\mathrm{MC}}$ is a van der Waals volume of one silver mercaptide particle, and $N$ is the number of SM particles in one cluster. The parameter 0.6 was selected to provide the approximate reproduction of the volume of SM cluster for $N=2-8$. For example, the diameter of the model cluster is $15.2 \AA$ for $N=4$. The average diameter of stable filament-like aggregates in the atomistic model is $\sim 15.5 \AA$. For the modeling of $-\mathrm{C}(\mathrm{O}) \mathrm{O}^{-}$and $-\mathrm{NH}_{3}{ }^{+}$functional groups, additional spherical centers of force are attached to the surface of particle of the type $A$. Further we will describe these centers as subsystems $B$ and $C$ (see Fig. 13). They carry 
negative and positive charges, respectively. For the sake of simplicity, we suppose centers of force $B$ and $C$ to have the same diameter $\sigma^{(\mathrm{NH} 3)}=\sigma^{(\mathrm{COO})} \equiv D$. Since SM particles in CSS are zwitterions, the number of positive and negative centers of force is equal to the number of SMs in each cluster. For simplicity, we assume centers of force $B$ and $C$ to be distributed over the surface randomly. Solvent is considered as a continuous medium with dielectric constant $\varepsilon$.

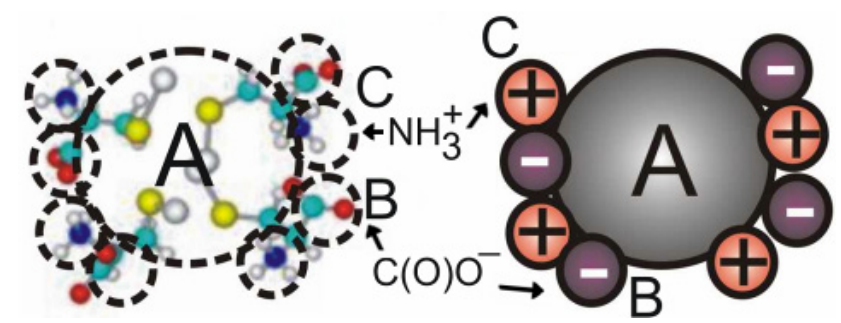

Figure 13. The mapping of an atomistic model of a cluster of SM particles onto the coarse grain representation.

The total number of SM clusters in the system is $N_{\mathrm{MC}}=$ $c_{0} C_{\mathrm{MC}} / N$. Here $C_{\mathrm{MC}}$ is the concentration of SM in the solution expressed as $\mathrm{mol} / \mathrm{L}, c_{0}=N_{\mathrm{A}} /\left(10^{9} l\right)^{3}$, where $N_{\mathrm{A}}$ is the Avogadro constant, while $l$ is the size of the simulation cell edge measured in Angstrom units. The total potential energy of the system is determined by the sum of three contributions, namely, excluded volume, screened Coulomb potential, and hydrogen bonds

$$
U=\sum_{i=1, i<j}^{N_{M C}(1+2 N)}\left(U_{i j}^{(e x c l)}+U_{i j}^{(Q)}+U_{i j}^{(H)}\right)
$$

that has the following analytical form (means that $\sigma_{\mathrm{ij}}=$ $\left.\left(\sigma_{\mathrm{i}}+\sigma_{\mathrm{j}}\right) / 2\right)$

$$
\begin{gathered}
U_{i j}^{(e x c l)}=\left\{\begin{array}{l}
E_{i j}\left(\left(\frac{\sigma_{i j}}{r_{i j}}\right)^{12}-\left(\frac{\sigma_{i j}}{r_{i j}}\right)^{6}+\frac{1}{4}\right), r_{i j} \leq 2^{1 / 6} \sigma_{i j} \\
0, r_{i j}>2^{1 / 6} \sigma_{i j}
\end{array},\right. \\
U_{i j}^{(Q)}=\frac{Z_{i} Z_{j} e^{2}}{4 \pi \varepsilon_{0} \varepsilon r_{i j}} e^{-\kappa r_{i j}}, \kappa=\sqrt{\frac{e^{2}}{\varepsilon \varepsilon_{0} k_{B} T} \sum_{\alpha} c_{\alpha} Z_{\alpha}^{2},} \\
U_{i j}^{(H)}=E_{i j}^{(H)}\left(\left(\frac{\sigma_{i j}}{r_{i j}}\right)^{12}-\left(\frac{\sigma_{i j}}{r_{i j}}\right)^{10}\right), r_{i j}<r_{c u t}^{(H)}
\end{gathered}
$$

The prime in the sum for total energy indicates that interactions between the centers of mesoscopic particles which belong to the same SM cluster are ignored, $\varepsilon_{0}$ is a permittivity of vacuum, $k_{\mathrm{B}}$ is the Boltzmann constant, $T=$
$300 \mathrm{~K}$. The value of all force constants equals to $E_{\mathrm{ij}}=1 k_{\mathrm{B}} T$, which is sufficient for the modeling of excluded volume of particles. The magnitude of dielectric constant $\varepsilon$ was fixed to be 79. To calculate $\kappa$, the concentrations of low-molecular-weight ions $\mathrm{C}_{\alpha}\left(\mathrm{H}_{3} \mathrm{O}^{+}, \mathrm{Ag}^{+}, \mathrm{NO}_{3}{ }^{-}, \mathrm{Na}^{+}, \mathrm{SO}_{4}{ }^{2-}\right)$ were determined with allowance made for CSS composition used in the construction of the atomistic model: $3.08,3.85$, and $0.2 \mathrm{mM}$ for L-cysteine, silver nitrate, and sodium sulfate, respectively.

Hydrogen bonds are supposed to arise only between the centers of particles of subsystems $\mathrm{B}$ and $\mathrm{C}$ of the neighboring clusters. The values of parameters $E_{\mathrm{ij}}{ }^{(H)}$ and $D$ were calculated by the fitting of the profile of potential energy $U_{\mathrm{ij}}^{(H)}$ (6) using calculated values of the total energy of two SM particles (interacting via $-\mathrm{C}(\mathrm{O}) \mathrm{O}^{-}$and $-\mathrm{NH}_{3}{ }^{+}$groups) after subtraction of the double energy of the single isolated particle. The calculations were made using Hartree-Fock approximation at the $6-31 \mathrm{G}^{* *}$ level. The best fit between calculated values and analytical representation for $U_{\mathrm{ij}}^{(H)}$ were obtained at $E_{\mathrm{ij}}^{\left({ }^{(H)}\right.}=86 \mathrm{kcal} / \mathrm{mol}$ and $D=2.8 \AA$. Cut-off radius $r_{\text {cut }}^{(H)}$ was established to be $3 D$.

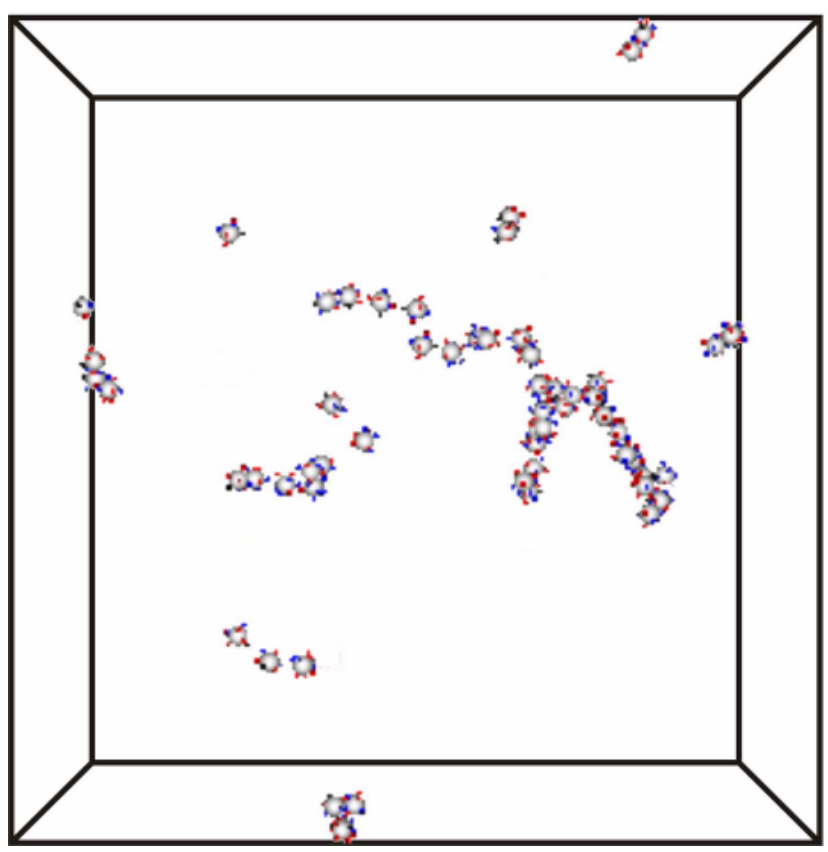

Figure 14. The snapshot of a simulation cell after $1 \cdot 10^{7} \mathrm{MC}$ steps. $N_{\mathrm{MC}}=$ $57, N=4, l=500 \AA$.

Modeling was carried out in a cubic cell with edge length $l$ of $500 \AA$. The number of SM clusters was calculated from the relationships between $N_{\mathrm{MC}}$ and $l$ described above. The evolution of CSS was performed by the Monte Carlo method. The sequence of microstates corresponding to the given canonical ensemble was generated by the essential sampling method [49]. Each new system configuration is built from the previous one by moving or rotating (with equal probability) of a randomly selected SM cluster. The initial coordinates of the clusters and their orientation were selected in a random manner. The probability $P$ of the emergence of a new configuration was assessed to be $P(\Delta U)=\min$ 
$\left\{1, \exp \left(\Delta U / k_{B} T\right)\right\}$, where $\Delta U$ is the difference between the total energy (3) of the system in some old and new state. The displacement of particles during modeling is smaller than 0.1 - $1 \AA$ and is selected so that the probability of the adoption of the new configuration is to be 0.5 . To exclude the effect of initial state on the final state of the system for each set of parameters, we performed a series of six independent calculations of $110^{7} \mathrm{MC}$ steps.

In our simulations, the number of particles in SM clusters was constant, and fixed $N=2,3$ and 4. Fig. 14 shows a snapshot of the CSS model after $10^{7} \mathrm{MC}$ steps of MC simulation. The emergence of a nucleation center of the filament-like aggregate and paired clusters is clearly seen. The calculation of aggregation number $N_{\mathrm{agg}}$ provides an opportunity for detailed monitoring of the evolution course. This value is equal to the number of aggregates composed of $L_{\text {agg }}$ SM clusters. Fig. 15 shows the growth of $N_{\text {agg }}$ with the number of modeling steps for the aggregates of different $L_{\text {agg. }}$. It is seen from the figure that the evolution of CSS is characterized by the gradual growth of the number of aggregates in solution and their total length. Thus, the developed mesoscopic model of CSS explains the formation of filament-like aggregates by means of the formation of noncovalent linking between the neighboring clusters of SM particles.

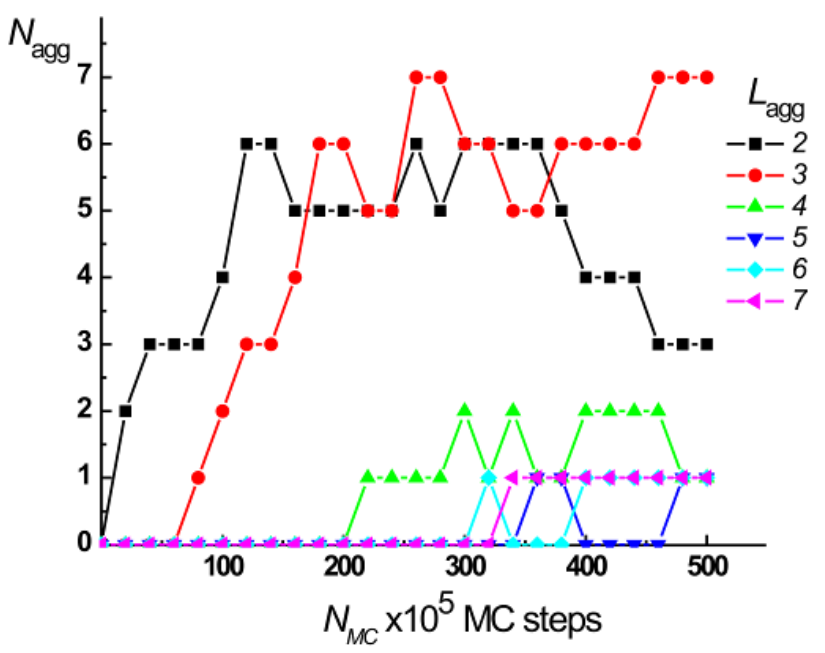

Figure 15. Example of change in the number of aggregates Nagg composed of Lagg SM particles depending on the number of the simulation steps, $\mathrm{NMC}=57, \mathrm{~N}=4$.

\section{Conclusions}

We have described a method for preparing a hydrogel based on aqueous solution of L-cysteine and silver nitrate. The main features of this hydrogel are self-assembly at low content of dispersed phase $(\sim 0.01 \%)$ and thixotropic behavior. The stage of CSS aging is accompanied by the appearance and growth of absorption bands at 310 and 390 $\mathrm{nm}$ in UV-vis spectra of the sample, that corresponds to the formation of aggregates of SM. Aggregation in CSS, leading to formation of molecular clusters, is confirmed by DLS. TEM showed that the addition of an electrolyte to CSS induces the formation of a supramolecular network and gelation of the solution. Experimental data allowed us to formulate a computer model used in the search for the mechanism of gelation in the system.

The developed atomistic model of aqueous solution of SM containing $\mathrm{AgNO}_{3}$ and $\mathrm{Na}_{2} \mathrm{SO}_{4}$ was used for studying molecular mechanisms of gelation. The model makes explicit allowance for the interaction between pairs of sulfur and silver atoms that belong to different SM particles. For the set of parameters used, we have built a series of initial configurations that differs in the ordering of SM viz. disordered, cluster, and filament-like structures. The analysis of the evolution course of prepared systems allowed us to monitor the formation of aggregates composed of SM particles and low-molecular-weight ions. A separate study was performed for filament-like aggregates repeated over periodic boundary conditions that presumably have to be the final stage of the evolution of the CSS model. The filament-like aggregates constructed on the basis of a chain of SM molecules (molecules are bound successively through noncovalent linking) show a tendency to degradation. Similarly to the evolution of the disordered and cluster initial configurations, the breaking of filament-like aggregates in the modeling cell leads to aggregates of extended shape, where the concentration of SM per unit volume is twice as high as in the initial filament-like state. Not all the new filament-like aggregates built up from the double number of SM molecules decomposed over the achieved modeling times. The analysis of the resulting molecular configurations shows that the filament-like aggregates are stabilized owing to the interaction of $-\mathrm{NH}_{3}{ }^{+}$and $-\mathrm{C}(\mathrm{O}) \mathrm{O}^{-}$groups that belong to SM particles within neighboring clusters. Such a principle of self-assembly is also described for other systems $[33,35,36,50,51]$, that indicates the universal character of the observed mechanism of self-assembly in CSS.

The data obtained on the cluster structure of filament-like aggregates and the features of their interaction provided a foundation for the mesoscopic model that allows evident demonstration of the emergence of gel network in CSS in a large scale.

In conclusion, it should be noted that the combination of experimental studies and computer modeling has allowed us to reveal the possible molecular mechanism of the formation of CSS gel network, which has a large applied significance for the understanding of gelation in other systems.

\section{Acknowledgements}

The work was supported by the Russian Ministry of Education "Nanostructured supramolecular hydrogels of bioactive substances for medical use" (Project No 3.18.41.2011) and "Design and development of a new type gel technology as the basis for creating ultra-high fiber and medicines" (Order No 2596 of 3.11.2011). The authors also 
thank the Shared Facilities Center of the Lomonosov Moscow State University, and the Joint Supercomputer Center, Russian Academy of Sciences (www.jscc.ru) for providing computing resources of the MVS-100k cluster.

\section{REFERENCES}

[1] P. Terech, R. G.Weiss. Low Molecular Mass Gelators of Organic Liquids and the Properties of Their Gels, Chem. Rev., Vol.97, No.8, 3133-3159, 1997.

[2] L. Estroff, A. D. Hamilton. Water Gelation by Small Organic Molecules, Chem. Rev., Vol.104, No.3, 1201-1217, 2004.

[3] M. Loos, B. L. Feringa, J. H. Esch. Design and Application of Self-Assembled Low Molecular Weight Hydrogels, Eur. J. Org. Chem., Vol.2005, No.17, 3615-3631, 2005.

[4] C. C. Lin, A. T. Metters. Hydrogels in controlled release formulations: Network design and mathematical modeling, Advanced Drug Delivery Reviews, Vol.58, No.12-13, 1379-1408, 2006.

[5] P. J. Flori. Introductory Lecture, Faraday Discuss, Chem. Soc., Vol.57, 7-18, 1974.

[6] A. Coniglio, H. E. Stanley, W. Klein. Site-Bond Correlated-Percolation Problem: A statistical Mechanical Model of Polymer Gelation, Phys. Rev. Lett., Vol.42, No.8, 518-522, 1979.

[7] D. Stauffer. Can percolation theory be applied to critical phenomena at gel points? Pure and Applied Chemistry, Vol.53, No.8, 1479-1487, 1981.

[8] D. Stauffer, A. Coniglio, M. Adam. Gelation and Critical Phenomena, Adv. Polym. Sci. Adv. Polym. Sci. Vol.44, 103-158, 1982.

[9] L. L. Vedenov, E. B. Levchenko. Supermolecular liquid-crystalline structures in solutions of amphiphilic molecules, Soviet Physics Uspekhi, Vol.26, No.9, 747-774, 1983.

[10] W. M. Gelbart, A. Ben-Shaul. The "New" Science of "Complex Fluids", J. Phys. Chem., Vol.100, No.31, 13196-13189, 1996.

[11] A. P. Philipse, A. M. Wierenga. On the Density and Structure Formation in Gels and Clusters of Colloidal Rods and Fibers, Langmuir, Vol.14, No.1, 49-54, 1998.

[12] J. H. van Esch, B. L. Feringa. New Functional materials Based on Self-Assembling Organogels: From Serendipity towards Design, Angew. Chem. Int. Ed., Vol.39, No.13, 2263-2266, 2000.

[13] J. H. Fuhrhop, W. Helfrich. Fiuld and Solid Fibers Made of Lipid Molecular Bilayers, Chem. Rev., Vol.93, No.4, 1565-1582, 1993

[14] A. N. Glebov, I. G. Grigor'eva. Gelatinizing of Aqueous Solutions of Transition-Metal Complexes, Russian Journal of Coordination Chemistry., Vol.28, No.5, 369-370, 2002.

[15] A. Ciferri (Ed.). Supramolecular Polymers, Dekker, New York, 2000.
[16] P. M. Pakhomov, M. M. Ovchinnikov, S. D. Khizhnyak, M. V. Lavrienko, W. Nierling, M. D. Lechner. Study of Gelation in Aqueous Solutions of Cysteine and Silver Nitrate, Colloid Journal, Vol.66, No.1, 65-70, 2004.

[17] G. R. Lenz, A. E. Martell. Metal Chelates of Some Sulfur-containing Amino Acids, Biochemistry, Vol.3, No.6, 745-750, 1964.

[18] M.-O.M. Piepenbrock, N. Clarke, J.W. Steed. Rheology and silver nanoparticle templating in a bis(urea) silver metallogel, Soft Matter, Vol.7, No.6, 2412-2418, 2011.

[19] L. Brunsveld, B. J. B.Folmer, E. W. Meijer, R. P. Sijbesma. Supramolecular Polymers, Chem. Rev., Vol.101, No.12, 4071-4097, 2001.

[20] M. J. Serpe, S. L. Craig. Physical Organic Chemistry of Supramolecular Polymers, Langmuir, Vol.23, No.4, 1626-1634, 2007.

[21] P. M. Pakhomov, S. S. Abramchuk, S. D. Khizhnyak, M. M. Ovchinnikov, V. M. Spiridonova. Formation of Nanostructured Hydrogels in L-cysteine and Silver Nitrate Solutions, Nanotechnologies in Russia, Vol.5, No.3-4, 209-213, 2010.

[22] S. Provencher. A Constrained Regularization Method for Inverting Data Represented by Linear Algebraic or Integral Equations, Comp. Phys. Comm., Vol.27, No.3, 213-227, 1992.

[23] W. Burchard. Dynamic Light Scattering Method of Polymer Solutions, Macromol. Symp. Vol.101, No.1, 103-113, 1996.

[24] H. B. Vickery, C. S. Leavenworth. The Behavior of Cystine with Silver Salts, J. Biol. Chem., Vol.86, No.1, 129-143, 1930.

[25] A. Barth. The Infrared Absorption of Amino Acid Side Chains, Progress in Biophysics and Molecular Biology, Vol.74, No.3-5, 141-173, 2000.

[26] Y. Gao, P. Jiang, L. Song, L. Liu, X. Yan, Z. Zhou, D. Liu, J. Wang, H. Yuan, Z. Zhang, X. Zhao, X. Dou, W. Zhou, G. Wang, S. Xie. Growth Mechanism of Silver Nanowires Synthesized by Polyvinylpyrrolidone-Assisted Polyol Reduction, J. Phys. D: Appl. Phys., Vol.38, No.7, 1061-1067, 2005.

[27] M. V. Varghese, R. S. Dhumal, S. S. Patil, A. R. Paradkar, P. K. Khanna, Synthesis and In-Vitro Antimycobacterial Studies of Cysteine Capped silver Nano-Particles, Reactivity in Inorganic, Metal-Organic, and Nano-Metal Chemistry, Vol.39, No.9, 554-558, 2009.

[28] L. O. Andersson. Study of Some Silver-Thiol Complexes and Polymers: Stoichiometry and Optical Effects, J. Polym. Sci. Part A-1: Polymer Chemistry, Vol.10, No.7, 1963-1973, 1972.

[29] R. A. Bell, J. R. Kramer. Structural Chemistry and Geochemistry of Silver-Sulfur Compounds: Critical Review, Environmental Toxicology and Chemistry, Vol.18, No.1, 9-22, 1999.

[30] G. Shramm. A practical approach to rheology and rheometry, Gebrueder: HAAKE, GmbH, Karlsruhe, 1994.

[31] W. Bae, R. K. Mehra. Cysteine-capped ZnS nanocrystallites: Preparation and characterization, Journal of Inorganic 
Biochemistry, Vol.70, No.2, 125-135, 1998.

[32] M. C. Brelle, J. Z. Zhang, L. Nguyen, R. K. J. Mehra. Synthesis and Ultrafast Study of Cysteine- and Glutathione-Capped $\mathrm{Ag}_{2} \mathrm{~S}$ Semiconductor Colloidal Nanoparticles, Phys. Chem. A., Vol.103, No.49, 10194-10201. 1999.

[33] S. Mandal, A. Gole, N. Lala, R. Gonnade, V. Ganvir, M. Sastry. Studies on the Reversible Aggregation of Cysteine-Capped Colloidal Silver Particles Interconnected via Hydrogen Bonds, Langmuir, Vol.17, No.20, 6262-6268, 2001.

[34] Ch. Barglik-Chory, Ch. Remenyi, H. Strohm, G. Mu1ller. Adjustment of the Band Gap Energies of Biostabilized CdS Nanoparticles by Application of Statistical Design of Experiments, J. Phys. Chem. B., Vol.108, No.23, 7637-7640, 2004.

[35] S. Panigrahi, S. Kundu, S. Basu, S. Praharaj, S. Jana, S. Pande, S. K. Ghosh, A. Pal, T. Pal. Cysteine functionalized copper organosol: synthesis, characterization and catalytic application, Nanotechnology, Vol.17, No.21, 5461-5468, 2006.

[36] B. Zhang, X. Ye, W. Hou, Y. Zhao, Y. Xie. Biomolecule-Assisted Synthesis and Electrochemical Hydrogen Storage of $\mathrm{Bi}_{2} \mathrm{~S}_{3}$ Flowerlike Patterns with Well-Aligned Nanorods, J. Phys. Chem. B., Vol.110, No.18, 8978-8985, 2006.

[37] J. Xiang, H. Cao, Q. Wu, S. Zhang, X. Zhang, A. A. R. Watt. L-Cysteine-Assisted Synthesis and Optical Properties of Ag2S Nanospheres, J. Phys. Chem. C., Vol.112, No.10, 3580-3584, 2008.

[38] N. W. H. Adams, J. R. Kramer. Potentiometric Determination of Silver Thiolate Formation Constants Using a Ag2S Electrode, Aqua. Geochem., Vol.5, No.1, 1-11, 1999.

[39] S. S. Voyutskii. Course of colloid chemistry, Khimiya, Moscow, 1976.

[40] W. D. Cornell, P. Cieplak, C. I. Bayly, I. R. Gould, K. M. Merz, Jr, D. M. Ferguson, D. C. Spellmeyer, T. Fox, J. W. Caldwell, P. A. Kollman. A Second Generation Force Field for the Simulation of Proteins, Nucleic Acids, and Organic Molecules, J. Am. Chem. Soc., Vol.117, No.19, 5179-5197,
1995.

[41] D. Case, T. Darden, T. Cheatham, C. Simmerling, J. Wang, R. Duke, R. Luo, M. Crowley, R. W. Zhang. Amber 10, University of California: San Francisco, USA 2008.

[42] W. L. Jorgensen, J. Chandrasekhar, J. D. Madura, R. W. Impey, M. L. Klein. Comparison of simple potential functions for simulating liquid water, J. Chem. Phys., Vol.79, No.2, 926-935, 1983.

[43] H. Sun. Ab Initio Calculations and Force Field Development for Computer Simulation of Polysilanes, Macromolecules, Vol.28, No.3, 701-712, 1995.

[44] R. C. Bingham, M. J. S. Dewar, D. H. Lo. Ground States of Molecules. XXV. MINDO/3. Improved Version of the MINDO Semiempirical SCF-MO Method, J. Am. Chem. Soc., Vol.97, No.6, 1285-1293, 1975.

[45] Molecular Modeling software, Hypercube Inc., Gainesville, FL, USA.

[46] V. M. Spiridonova, V. S. Savel'eva, S. D. Khizhnyak, M. M. Ovchinnikov, P. M. Pakhomov. Rheological Tests of Cysteine-Silver Solution and the Hydrogel on the Basis Thereof, Tver State University, Fiziko-Khimiya Polymerov, No.15, 88-93, 2009.

[47] W. Smith, T. R. Forester. DL_POLY_2.0: A general-purpose parallel molecular dynamics simulation package, J. Mol. Graph., Vol.14, No.3, 136-141, 1996.

[48] M. P. Allen, D. J. Tildesley. Computer Simulation of Liquids, Clarendon Press, Oxford, 1989.

[49] N. Metropolis, A. W. Rosenbluth, M. N. Rosenbluth, A. N. Teller, E. Teller. Equation of State Calculations by Fast Computing Machines, J. Chem. Phys., Vol.21, No.6, 1087-1092, 1953.

[50] A. Yu. Men'shikova. Monodisperse Functional Polymeric Particles and Their Application for Nanotechnology, Nanotechnologies in Russia, Vol.5, No1-2, 35-48, 2010.

[51] P. K. Sukul, S. Malik. Supramolecular Hydrogels of Adenine: Morphological, Structural and Rheological Investigations, Soft Matter, Vol.7, No.9, 4234-4241, 2011. 